\title{
WDM BASED FSO LINK OPTIMIZING FOR 180KM USING BESSEL
}

\section{FILTER}

\author{
Bikram Beri $^{1}$, Neel Kamal ${ }^{2}$ \\ ${ }^{1,2}$ Department of Electronic and Communication Engineering in GTBKIET Chhapianwali, Malout, India
}

\begin{abstract}
Free space optical link is a growing field in communication due to its advantage of wide bandwidth, high security and easy installation. A wavelength division multiplexing (WDM) access network using free space optical (FSO) communication in different weather conditions like haze and rain are discussed in this article and find out the possibility of communication link up to $180 \mathrm{~km}$ in clear weather with $2.5 \mathrm{Gbps}$ data rate on the wavelength of $1550 \mathrm{~nm}$ and up to $54 \mathrm{~km}$ in haze condition using same data rate \& wave length. Further the effect of using two different low pass filter (Gaussian and Bessel) at the receiver are discussed and conclude that Bessel filter is better on 2.5 Gbps data rate for WDM based FSO link.
\end{abstract}

Keywords: optical communications, wavelength Division Multiplexing (WDM), free space optics (FSO)

\section{INTRODUCTION}

Today is the world of high data rate communication. From the last few decade communication between devices gone to their extreme speed with the use of wired link and wireless RF link but these transmitting media have limited band width, so to enhance the band width optical fiber is to be utilized for all most infinity band width but OFC is a cost effective media due to the use of splicing on joint it is not possible to provide the optical link in terrestrial network (in metro political area) so to provide the high band width link between devices in metro political area , communication link required wireless link. So to full fill the requirement of wide band width wireless link free space optics (FSO) communication media is to be find out. In free space optics communication system very high band width wireless link can be established. It is very much efficient in indoor or obstacle less place where line of site communication can be possible without the turbulence environment but in outdoor communication there is environmental effect like fog, haze, rain and dust which limits the FSO link .The combination of environmental attenuation and earth inequalities losses provide total attenuation. Signal diverts from its actual path due to scattering caused by different earth unequal points. Environmental conditions like rain and snow fall causes affect similar to Rayleigh scattering because particles of rain and snow are so big. Differential heating can produce turbulence in random fluctuation for the refractive index of air due to that defocusing of beam is generated that produces received signal intensity vacillation and spread the transmitting beam .For better communication we also need large link distance, less scattering, less absorption, less misaiming, large laser power \& data bits. We also have to make a reduced cost, faster implementation and easy installable over long distances. To get these and to reduce the effect of these environmental condition we need a special quality of FSO link .To do so WDM based FSO link can be used for long range and with wide data rate of 2.5 GBPS .For it we have to use the frequency range of $1550 \mathrm{~nm}$ with high power laser, a suitable receiver aperture, highly sensitive light detector and better modulation technique is to improve the system performance in term of quality of the transmission.

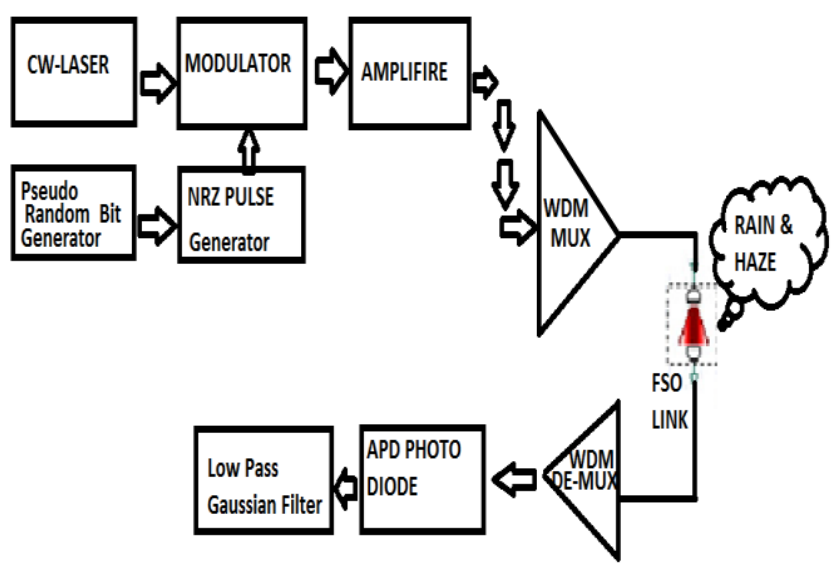

Fig.1:-WDM base FSO Communication Link Block Diagram

Table 1: Basic characteristic and data observation

\begin{tabular}{lll}
\hline Characteristic & \multicolumn{1}{c}{ Data } & Observation \\
\hline Data rate & $2.5 \mathrm{Gbps}$ & Data rate Effect BER \\
Power & $10 \mathrm{db}$ & Power also effect BER \\
Link range & $180 \mathrm{Km}$ & Also Effect BER \\
Receiver & $15 \mathrm{~cm}$ & Also Effect BER \\
Aperture & & \\
No of user & Depend & No of User versus BER \\
\hline
\end{tabular}




\section{SYSTEM ANALYSIS}

\subsection{WDM System Design}

The basic diagram of WDM based FSO system is as shown in fig-1, in this block diagram is divided in three parts one is transmitter, second is receiver and third part is FSO link. In transmitter part we are using a CW laser with different power according to environment condition, Mach-Zehnder modulator for modulation, Pseudo-Random bit generator for carrier and NRZ Pulse Generator for encryption technique. In long distance communication we also need an optical amplifier. Whereas in receiver part to detect the optical signal we use APD Photodiode, low pass Bessel filter and for measuring output some tools such as Optical Time Domain Visualize and bit error rate visualize are used to visualize the result. In channel, WDM (Multiplexer and De-Multiplexer) technique and a FSO Path link is used. The other parameter like data rate frequency used and link range are shown in table-1; by using best parameter it is possible to make long range FSO link. The received signal quality is very much dependent on the natural environmental effect and design of WDM system.

\subsection{Link Margin Analysis}

The design receiver should have minimum sensitivity at the given data rate, so that the received power can be sufficient after receiving because received signal comes after FSO link And FSO link have the losses due to media (air)

Table-2

\begin{tabular}{|l|l|}
\hline \multicolumn{2}{|l|}{ FSO link parameters, constant value } \\
\hline Gravitational Constant & $980 \mathrm{~cm} / \mathrm{s}^{2}$ \\
\hline Water density & $1 \mathrm{~g} / \mathrm{cm}^{2}$ \\
\hline Viscosity of air & $1.8 \times 10^{-4}(\mathrm{~g} / \mathrm{cm})$ \\
\hline Droplet, a & $.001-01 \mathrm{~cm}$ \\
\hline Wavelength & $1550 \mathrm{~nm}$ \\
\hline$Q_{\text {scat }}$ & 2 \\
\hline
\end{tabular}

\begin{tabular}{|l|l|}
\hline \multicolumn{2}{|l|}{ Rain Fall Rate } \\
\hline Type & $\mathrm{Cm} / \mathrm{s}$ \\
\hline Light & $7.22 \times 10^{-4}$ \\
\hline Medium & $1.11 \times 10^{-3}$ \\
\hline Heavy & $2.22 \times 10^{-3}$ \\
\hline
\end{tabular}

between transmitter and receiver and it is also changing time to time due to different weather condition. So we have to find out the effect of weather conditions such as, rain and haze losses on FSO link.

\subsection{Rain}

Rain is the highest attenuation factor in environment for light that's why it also affects the FSO link very much. Rain can reduce the visibility to much higher intensity so we have to find out the effect of rain on FSO link .For local rain data mathematic model is derived as given below. Beer's and Stroke's law represent the mathematical equation for it. Beer's law describe laser power attenuation in the environment $[4,8]$ : It represents that

$$
\mathbf{T}(\mathbf{R})=\mathbf{P}(\mathbf{R}) / \mathbf{P}(\mathbf{O})=\mathrm{e}^{-\beta R} \quad \ldots .1
$$

All parameter in this equation are as given below:

$\mathrm{R}=$ link range (meters)

$\mathrm{T}(\mathrm{R})=$ transmittance in the range $\mathrm{R}(\mathrm{km})$

$\mathrm{P}(\mathrm{R})$ =laser power in range $\mathrm{R}$

$\mathrm{P}(\mathrm{O})=$ laser power at source

$\mathrm{B}=$ scattering coefficient $\left(\mathrm{Km}^{-1}\right)$

The laser light is very much affected by rain drop, haze, clouds and heavy fog etc. All these parameter can scatter the light beam produced by laser, it is not possible to find out the correct rate of scattering beam the attenuation coefficient is not dependent on the laser wavelength. Stroke' law can be used to calculate the scattering coefficient of beam due to rain drop or fog. It is as given below;

$$
\beta_{\text {rain scat }}=\pi \mathrm{a}^{2} \mathrm{NaQ}_{\text {scat }}(\mathrm{a} / \lambda)
$$

Here ' $a$ ' represent radius of raindrop $(0.001-0.1 \mathrm{~cm})$, ' $\mathrm{Na}$ ' represent rain drop distribution. ' $Q_{\text {scat }}$ ' represent scattering efficiency, $\lambda$ represent wavelength of the rain drop distribution in this equation, by using equation (1 and 2) $\mathrm{Na}$ can be calculated.

\section{$\mathrm{Na}=\mathrm{Za} /\left[4 / 3\left(\pi \mathrm{a}^{3}\right) \mathrm{VA}\right.$}

Here $\mathrm{Za}$ represent the rainfall rate $(\mathrm{cm} / \mathrm{s})$, ' $\mathrm{a}$ ' represent droplet radius and $\mathrm{Va}$ represent limit speed precipitation The raindrop Limiting speed is also given by

$$
\mathrm{VA}=\left(2 \mathrm{a}^{2} \rho g\right) / 9 \eta
$$

In it $\rho$ represent water density $\left(\mathrm{g} / \mathrm{cm}^{2}\right)$, ' $\mathrm{g}$ ' represent gravitational constant and $\eta$ represent air viscosity. The constant value of these parameters is given by Table-2 [1] Attenuation of light rain is recorded for rain is $6.27 \mathrm{~dB} / \mathrm{km}$, for medium rain $9.64 \mathrm{~dB} / \mathrm{km}$ while for heavy rain 19.28 $\mathrm{dB} / \mathrm{km}$ at $1550 \mathrm{~nm}$ wavelength.[1] 


\subsection{Haze}

As we see in our environment rainy season or rain time period is small but the haze remains for longer time in the environment, so affect of haze is more on FSO link, so it is very important to find out the effect of haze on FSO link . The measurement of FSO parameter can be done by measuring the actual system [6] but this experiment is not efficient every time so we have to find out the another method to find forecast the system performance[8].

The mathematical models for it is represented by Kim \& Kruse as given below

$$
\beta=(3.91 / V)(\lambda / 550 \mathrm{~nm})^{-q}
$$

Where, $\beta$ represent haze attenuation, $\mathrm{V}$ represents visibility in kilo meters, $\lambda$ represent wavelength in nanometres and $q$ represent the size of distribution so the attenuation constant for haze condition is given by 0.23 $\mathrm{dB} / \mathrm{km}$ at $1550 \mathrm{~nm}$ frequency for the visibility range $(6 \mathrm{~km}<$ $\mathrm{V}<50 \mathrm{~km}$ ) and at heavy haze $2.37 \mathrm{db} / \mathrm{km}$ at $1550 \mathrm{~nm}$ for low visibility range $(\mathrm{V}<6 \mathrm{~km}):[1]$

\subsection{FSO Parameters Optimization}

FSO link is so much dependent on number of parameters like optical amplifier gain, laser power, data bit rate, receiver aperture, frequency used, and type of filter used etc. priority wise arrangement according to effect is as given in Fig-2. By using all these parameter the performance analyzed as given in Table-3 this formed for $180 \mathrm{~km}$ FSO link using beam spreading zero and also gives the comparative result of using Bessel low pass filter and Gaussian low pass filter in FSO receiver

Table-3: Optimization for the link of 180km

\begin{tabular}{|c|c|c|c|c|c|c|c|}
\hline $\begin{array}{c}\text { Weather } \\
\text { Condition }\end{array}$ & $\begin{array}{l}\text { Attenuation(db } \\
\text { ) }\end{array}$ & Bit Rate & $\begin{array}{c}\text { Laser } \\
\text { Power }\end{array}$ & $\begin{array}{c}\text { Responsivity } \\
\text { of APD }\end{array}$ & $\begin{array}{c}\text { Amplifier } \\
\text { Gain }\end{array}$ & BER(Bessel) & BER(Gaussian) \\
\hline Very clear & 0.065 & $2.5 \mathrm{Gbps}$ & $10 \mathrm{~dB}$ & 1 & No & 0 & 0 \\
\hline Clear & 0.233 & $2.5 \mathrm{Gbps}$ & $10 \mathrm{~dB}$ & 1 & No & $3.41661 \mathrm{e}-012$ & $7.37257 \mathrm{e}-012$ \\
\hline Light haze & 0.55 & $2.5 \mathrm{Gbps}$ & $10 \mathrm{~dB}$ & 10 & $50 \mathrm{db}$ & $1.55914 \mathrm{e}-040$ & $2.1429 \mathrm{e}-039$ \\
\hline Haze & \multicolumn{7}{|c|}{ Special cases } \\
\hline Rain & \multicolumn{7}{|c|}{ Special cases } \\
\hline
\end{tabular}

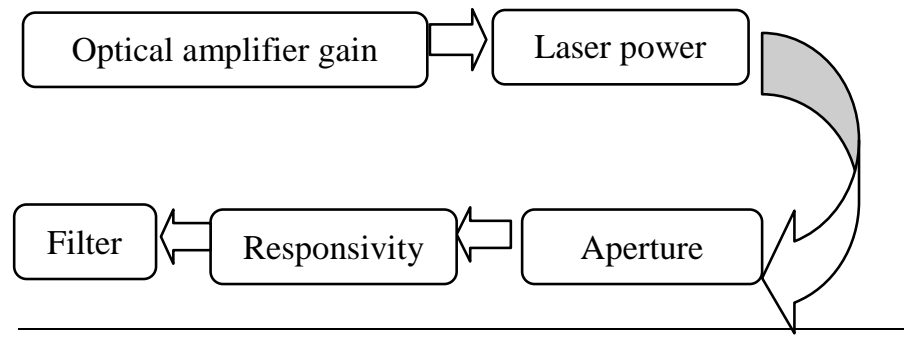

Fig 2: Priority wise Effect of parameter of WDM FSO system

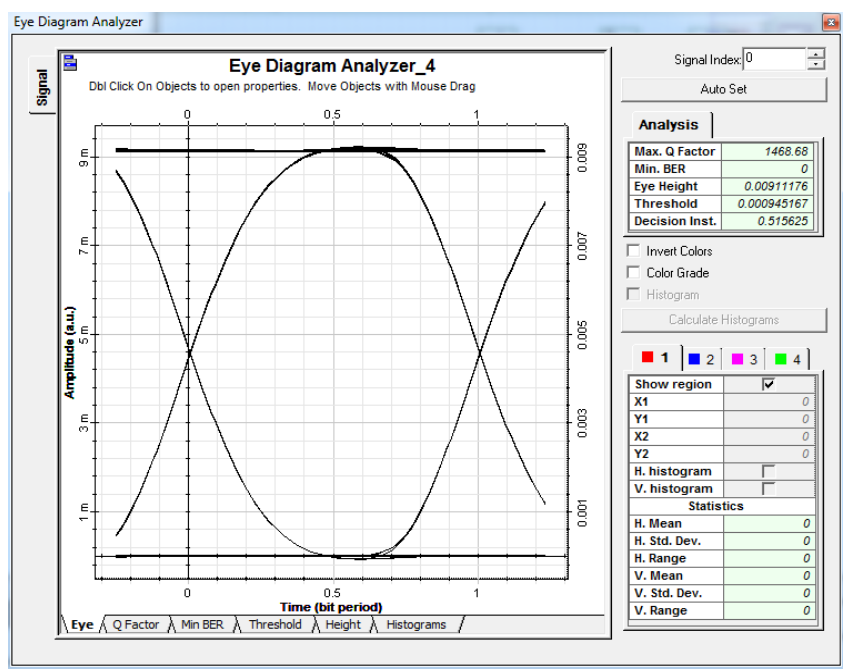

Fig 3: At very clear weather after $180 \mathrm{~km}$ 


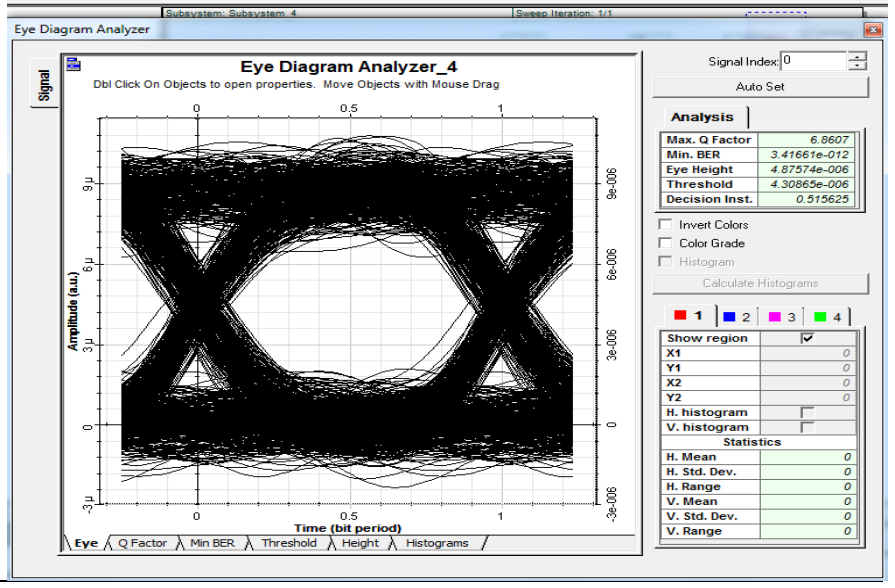

Fig-4: At clear weather after $180 \mathrm{~km}$ using Bessel filter

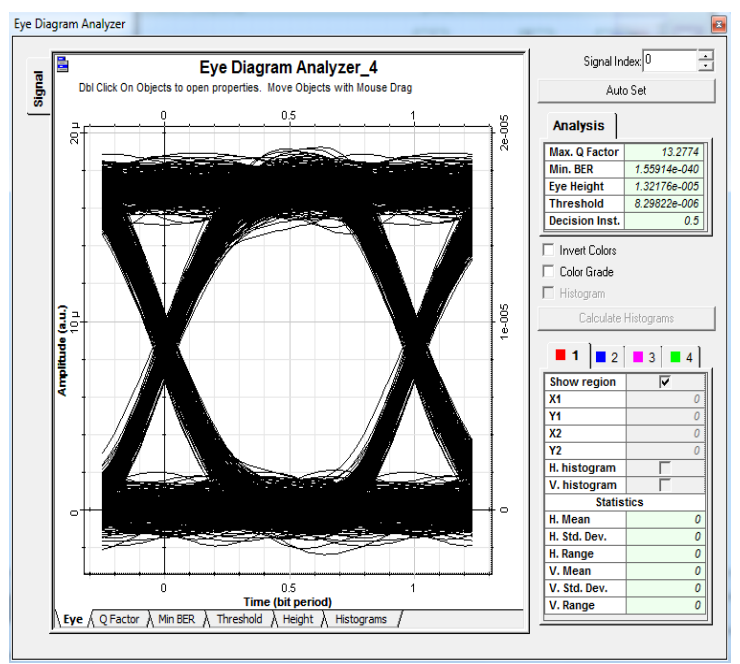

Fig-5: At light haze after $180 \mathrm{KM}$ using Bessel filter

During haze and rain visibility reduces and it is not possible for the laser beam to pass this effect up to the $180 \mathrm{~km}$ but this condition appear come rarely. In routine days clear weather is present or some time light haze is there so in all these condition it is possible to make the WDM based FSO link or pass $2.5 \mathrm{Gbps}$ bit rate as shown in the Table 3 or fig.no.4, 5, 6 in this table one more thing is clear that if we are using Bessel filter rather than Gaussian filter, Bessel gives the better BER result so use of Bessel filter in WDM based FSO system give better results. In case of heavy haze or rain we have to make some improvement in our system because rain is the biggest problem in free space optics communication so for it we have to increase the power of the laser to $30 \mathrm{db}$, increase the gain of the amplifier to $50 \mathrm{db}$, receiver aperture to $30 \mathrm{~cm}$ and Responsivity of the APD photo diode to 10 in all cases we are using beam spreading zero table-4 and fig.6, 7, 8, 9can represent the different parameter result.

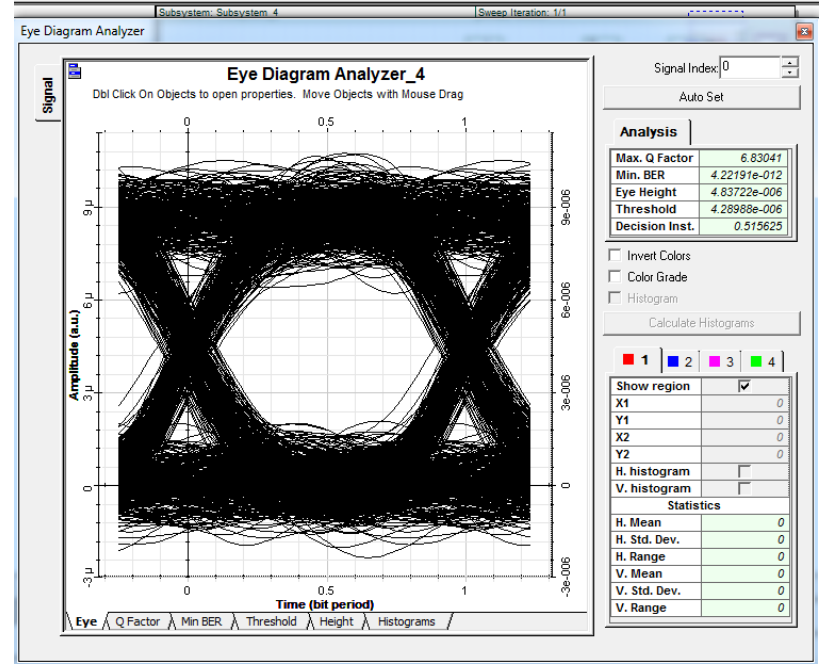

Fig-6: At heavy haze after $54 \mathrm{~km}$ using Bessel

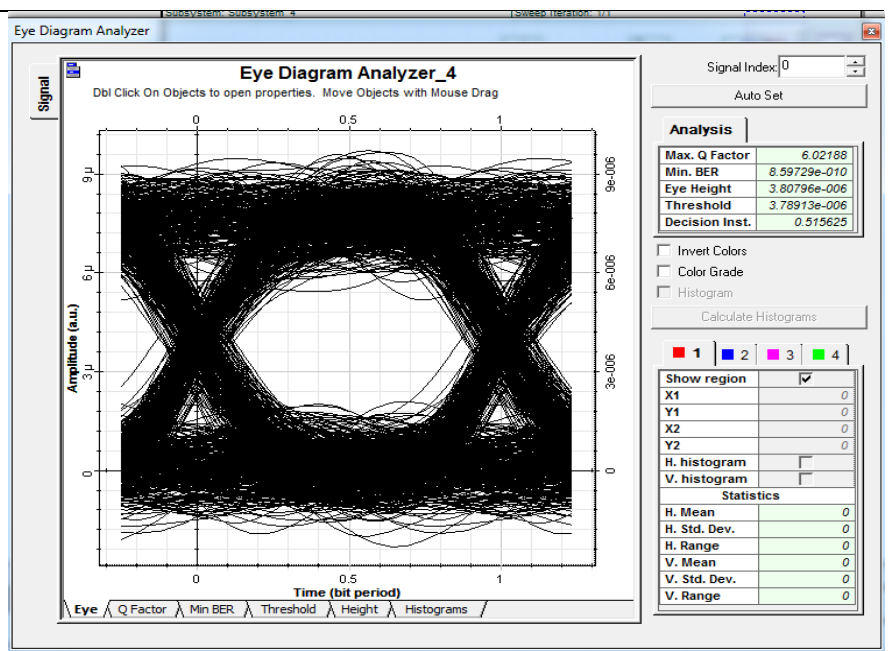

Fig-7: At light rain after $20.5 \mathrm{Km}$ using Bessel filter 
Table-4 Speatial case link analises

\begin{tabular}{|l|l|l|l|l|l|l|l|}
\hline $\begin{array}{l}\text { Weather } \\
\text { condition }\end{array}$ & $\begin{array}{l}\text { Attenuation } \\
(\mathbf{d B})\end{array}$ & $\begin{array}{l}\text { Bit Rate } \\
(\mathbf{G b p s})\end{array}$ & $\begin{array}{l}\text { Laser } \\
\text { power( } \\
\mathbf{d B})\end{array}$ & $\begin{array}{l}\text { Amplifier } \\
\text { Gain }\end{array}$ & BER(Bessel) & BER(Gaussian) & $\begin{array}{l}\text { Link range } \\
(\mathbf{k m})\end{array}$ \\
\hline $\begin{array}{l}\text { Heavy } \\
\text { haze }\end{array}$ & 2.37 & 2.5 & 30 & 50 & $4.22191 \mathrm{e}-012$ & $9.04123 \mathrm{e}-012$ & $54 \mathrm{~km}$ \\
\hline Light rain & $6.27 \mathrm{db}$ & 2.5 & 30 & 50 & $8.59729 \mathrm{e}-010$ & $1.56355 \mathrm{e}-009$ & $20.5 \mathrm{~km}$ \\
\hline $\begin{array}{l}\text { Medium } \\
\text { rain }\end{array}$ & $9.64 \mathrm{bd}$ & 2.5 & 30 & 50 & $9.70463 \mathrm{e}-008$ & $1.52509 \mathrm{e}-007$ & $13.4 \mathrm{~km}$ \\
\hline $\begin{array}{l}\text { Heavy } \\
\text { Rain }\end{array}$ & 19.28 & 2.5 & 30 & 50 & $9.70463 \mathrm{e}-008$ & $1.52509 \mathrm{e}-007$ & $6.7 \mathrm{~km}$ \\
\hline
\end{tabular}

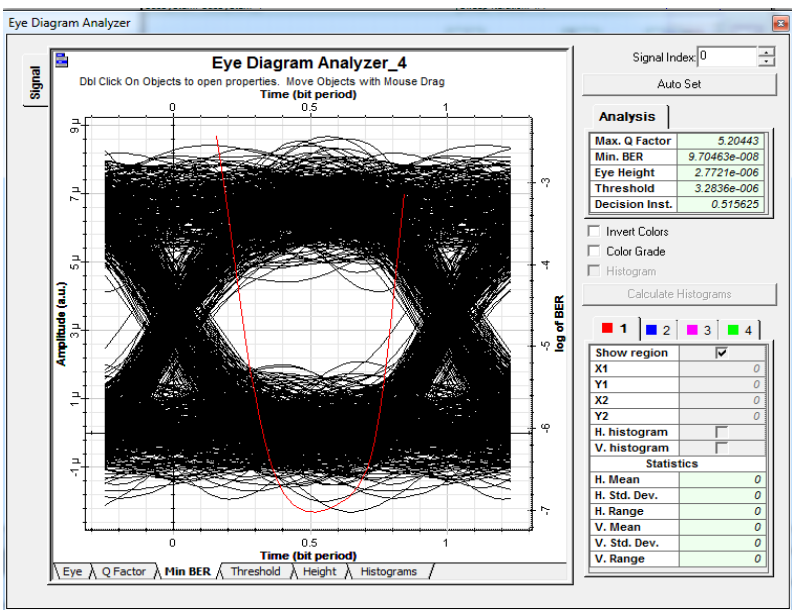

Fig-8: At medium rain after $13.4 \mathrm{Km}$ using Bessel filter

In this Table as we see that in heavy haze it is possible to communicate up to 54 kilo meter with a very good BER of 4.22191e-012 using $2.5 \mathrm{Gbps}$ data rate with wave length of $1550 \mathrm{~nm}$ shown in fig. 5 and is possible to increase the communication length up to $59 \mathrm{~km}$ with efficient data rate but in rain the communication decreases to 20.5 kilo meter with BER $8.59729 \mathrm{e}-010$ On $1550 \mathrm{~nm}$ wavelength or $2.5 \mathrm{Gbps}$ bit rate fig. 6 and 7 represent and decreases up to 6.7 kilo meter in heavy rain as shown in fig 8 and 9 . We can also improve these results by using number of Amplifier in transmitter and receiver or increasing the receiver aperture and laser power. All the above result represent the possibility of WDM based FSO communication in all the environment condition

\section{CONCLUSIONS}

In this paper simulation modelling of a WDM FSO system is investigated and found successful establishment of communication link. In this we are finding out that in clear weather condition communication link of $180 \mathrm{~km}$ with 2.5 Gbps data rate has been successfully transmitted through WDM FSO system.

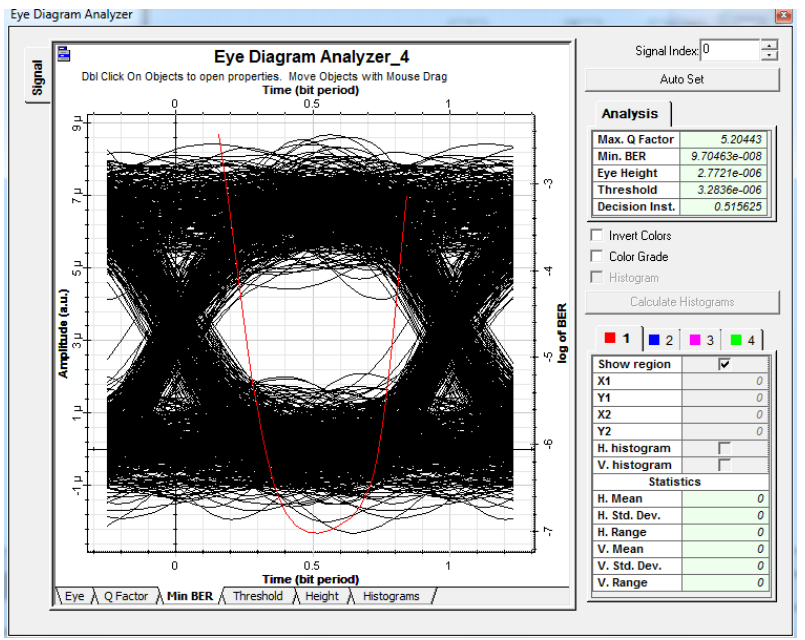

Fig-9: At heavy rain after 6.7 Km using Bessel filter

The simulation results indicate the trade-off between simulation parameters (link range, receiver aperture and input power).For example, at $2.5 \mathrm{Gbps}$ under clear weather, the BER value of 3.41661e-012 is achieved for $180 \mathrm{~km}$, while at $30 \mathrm{db}$ laser power, $30 \mathrm{~cm}$ aperture the BER value of $1.815230-021$ is achieved for $300 \mathrm{~km}$ transmission distance. The effect of filter used in receiver and environmental effect on FSO link has been presented experimentally (using OptiSystem version 10.0) and illustrates that Bessel filter having better result than Gaussian filter in $2.5 \mathrm{Gbps}$ bit rate in all condition like in rain, heavy haze and clear weather. In FSO link environmental parameter like longer links, heavy haze, light rain, medium rain and heavy rain generate highest attenuation so due to it to tackle this attenuation short link range can be used to optimize the FSO system. 


\section{REFERENCES:}

[1]. Hilal A. Fadhil a,*, Angela Amphawan b, Hasrul A.B. Shamsuddin a, Thanaa Hussein Abd a, Hamza M.R. Al-Khafaji a, S.A. Aljunid a, Nasim Ahmed Optimization of free space optics parameters: An optimum solution for bad weather conditions in: G Model IJLEO-52845@ 2013 Elsevier GmbH.

[2]. Xiaoming Zhu and Joseph M. Kahn, Free-Space Optical Communication Through

Atmospheric Turbulence Channels in: IEEE TRANSACTIONS ON COMMUNICATIONS, VOL. 50, NO. 8, AUGUST 2002

[3]. Mohamed1, Ahmed Nabih Zaki Rashed2*, and Amina E. M. El-Nabawy ; The Effects of the Bad Weather on the Transmission and Performance Efficiency of Optical Wireless Communication Systems in: I.J. Image, Graphics and Signal Processing, 2012, 7, 68- 83/ 10.5815/ijigsp.2012.07.08

[4]. I. Kim, E. Korevaar, Availability of free space optics (FSO) and hybrid FSO/RF systems", in: Proc. SPIE Optical Wireless Communication IV, vol. 530, Denver CO, August 21-22, 2001, pp. 84-95.

[5]. Nik Shahidah Afifi bt. Md. Taujuddin, Maisara Othman and Zarina Tukiran; Optimization for the Best Performance for Wavelength Division Multiplexed Passive

Optical Network Proceedings of EnCon2008 2nd Engineering Conference on Sustainable Engineering Infrastructures Development \& Management December 18 -19, 2008, Kuching, Sarawak, Malaysia ENCON 2008-F-28

[6]. M. Al Naboulsi, H. Sizun, F. de Fornel, Fog attenuation prediction for optical and infrared waves, Optic. Eng. 43 (2 (February)) (2004) 319-329.

[7]. Kulwant Singh, Baljeet Kaur; Study of WDM based FSO system for different weather conditions in: ICECE-04638(Accepted/Publication Proceedings), ICECE-2012, April $06-07,2012$.

[8]. H. Willebrand, S.B. Ghuman, Free-Space Optics: Enabling Optical Connectivity in Today's Network, Sams Publishing, Indiana 46240, USA, 2002 